\title{
On maximal functions of fractional order
}

G. O. OKIKIOLU (Norwich)

1. Introduction. Given any measurable function $f$ on $(0, \infty)$ we define the maximal function $M(f)$ associated with $f$ by

$$
M(f)(x)=\sup _{0 \leqslant t<x}\left|(x-t)^{-1} \int_{t}^{x} f(y) d y\right| \quad(x>0) .
$$

In this paper we consider certain extensions of $M(f)$ which we call maximal functions of fractional order. The simplest case of this is defined for measurable functions on $(0, \infty)$ by

$$
M_{r}(f)(x)=\left(\int_{0}^{x}\left|(x-t)^{-1} \int_{t}^{x} f(y) d y\right|^{r} d t\right)^{1 / r} \quad(x>0)
$$

It is obvious that $M(f)$ corresponds to $M_{\infty}(f)$. We shall call $M_{r}(f)$ the fractional maximal function of order $1 / r$.

The well-known theorem of Hardy and Littlewood [2] states that if $f \in L^{p}(0, \infty)$, then

$$
\|f\|_{p} \leqslant\|M(f)\|_{p} \leqslant p^{\prime}\|f\|_{p} \quad(1<p \leqslant \infty)
$$

where $(1 / p)+\left(1 / p^{\prime}\right)=1$, and

$$
\|f\|_{p}=\left(\int_{-\infty}^{\infty}|f(t)|^{p} d t\right)^{1 / p}
$$

Here we set $f(t)=0$ for $t \leqslant 0$ if it is defined only on the half-line $(0, \infty)$. One of the main results involving $M_{r}(f)$ shows that, for $r \geqslant p$, $(1 / q)=(1 / p)-(1 / r)$, we have

$$
\left\|M_{r}(f)\right\|_{q} \leqslant p^{\prime}\|f\|_{p}
$$

There are also some similar and simpler results in the case $0<r<p$. In section 6 we consider an extension of $M_{r}(f)$ defined on $(-\infty, \infty)$ by

$$
\tilde{N}_{r}(f)(x)=\left\{\int_{-\infty}^{\infty}\left|t^{-1} \int_{x-t}^{x} f(y) d y\right|^{r} d t\right\}^{1 / r}=\left\{\int_{-\infty}^{\infty}\left|\frac{F(x)-F(x-t)}{t}\right|^{r} d t\right\}^{1 / r},
$$


where $F(x)=\int_{0}^{x} f(y) d y$. Following this we consider fractional maximal functions defined on Euclidean space of $n$ dimensions. The last section of the paper is devoted to applications of results involving fractional maximal functions in the treatment of some integral operators.

2. Preliminary results. In this section we obtain some elementary results involving $M_{r}(f)$, and state preliminary results to be applied later. We shall assume throughout that $f$ is some measurable function on $(0, \infty)$.

(2.1) For $s \geqslant r>0$, we have

$$
x^{-1 / r} M_{r}(f)(x) \leqslant x^{-1 / s} M_{s}(f)(x) \quad(x>0) .
$$

Proof. This is easily verified by applying Hölder's inequality to the expression

$$
M_{r}(f)(x)^{r}=\int_{0}^{x}\left|(x-t)^{-1} \int_{t}^{x} f(y) d y\right|^{s(r / 8)} d t .
$$

(2.2) Let $0 \leqslant \alpha \leqslant 1,(1 / r)=\left(\alpha / r_{1}\right)+(1-\alpha) / r_{2}, 0<r_{1}, r_{2} \leqslant \infty$. Then

$$
M_{r}(f) \leqslant M_{r_{1}}(f)^{\alpha} M_{r_{2}}(f)^{1-\alpha}
$$

Proof. This easily follows by applying Hölder's inequality to the expression

$$
M_{r}(f)(x)^{r}=\int_{0}^{x}\left|(x-t)^{-1} \int_{t}^{x} f(y) d y\right|^{\mid r_{1}}\left|(x-t)^{-1} \int_{t}^{x} f(y) d y\right|^{(1-a) r_{2}} d t
$$

where $a=(\alpha r) / r_{1},(1-a)=(1-\alpha) r / r_{2}$.

(2.2.1) Note. The conclusion of 2.2 remains valid if $M_{r}(f)$ is replaced by any of the maximal functions considered in this paper.

(2.3) LEMMA. If $f \geqslant 0$ is in $L^{1}(0, b), 0<b<\infty$, and if $r>1$, then for almost all $x$ in $(0, b)$, we have

$$
\begin{aligned}
M_{r}(f)(x)^{r}= & r^{\prime} \int_{0}^{x} f(t)\left((x-t)^{-1} \int_{i}^{x} f(y) d y\right)^{r-1} d t-\left(r^{\prime} / r\right) x^{1-r}\left(\int_{0}^{x} f(y) d y\right)^{r}, \\
& M_{r}(f)(x) \leqslant r^{\prime}\left(\int_{0}^{x} f(t)^{r} d t\right)^{1 / r} \quad\left(f \in L^{r}(0, b)\right) .
\end{aligned}
$$

Proof. On integrating the left side of (i) by parts, we have

$$
\begin{aligned}
& \int_{0}^{x}\left((x-t)^{-1} \int_{t}^{x} f(y) d y\right)^{r} d t \\
= & {\left[(r-1)^{-1}(x-t)^{1-r}\left(\int_{t}^{x} f(y) d y\right)^{r}\right]_{0}^{x}+(r /(r-1)) \int_{0}^{x}(x-t)^{1-r}\left(\int_{t}^{x} f(y) d y\right)^{r-1} f(t) d t, }
\end{aligned}
$$

and the result (i) is easily verified since

$$
\lim _{t \rightarrow x}(x-t)^{-1} \int_{t}^{x} f(y) d y
$$

is finite for almost all $x$ in $(0, b)$

It is easily seen by applying Hölder's inequality to the right side of (i) that

$$
\begin{aligned}
M_{r}(f)(x)^{r} & \leqslant r^{\prime} \int_{0}^{x} f(t)\left((x-t)^{-1} \int_{t}^{x} f(y) d y\right)^{r-1} d t \\
& \leqslant r^{\prime}\left(\int_{0}^{x} f(y)^{r} d y\right)^{1 / r} M_{r}(f)(x)^{r-1}
\end{aligned}
$$

and this implies (ii).

In the sequel we shall frequently refer to the following extension of Hardy's inequality:

(2.4) LEMma. Let $f \in L^{p}(0, \infty), p>1,0 \leqslant \gamma \leqslant(1 / p),(1 / q)=(1 / p)-\gamma$ and let

$$
A(f)(x)=x^{-1} \int_{0}^{x} f(y) d y
$$

Then

$$
\left\|x^{\gamma} A(f)(x)\right\|_{q} \leqslant k_{p, \gamma}\|f\|_{p},
$$

where $k_{p, \gamma}=\left\{(1-\gamma) p^{\prime}\right\}^{(1-\gamma)}$.

Proof. In the ease $p>1,0 \leqslant \gamma<(1 / p)$, the lemma is proved in [5], Theorem 7 . The case $\gamma=(1 / p)$ is easily verified by applying Hölder's inequality.

3. The function $M_{r}(f): 0<r<p$. The three theorems that follow involve the cases $0<r<1, r=1,1<r<p$ respectively.

(3.1) THEOREM. Let $f \in L^{p}(0, \infty), 0 \leqslant \gamma \leqslant(1 / p)<1,0<r<1,(1 / s)$ $=(1 / p)-\gamma$. Then

$$
\begin{aligned}
& M_{r}(f)(x) \leqslant(1-r)^{-1 / r} x^{(1 / r)-1} \int_{0}^{x}|f(y)| d y, \\
& \left\|x^{\gamma-(1 / r)} M_{r}(f)(x)\right\|_{s} \leqslant(1-r)^{-1 / r} k_{p, \gamma}\|f\|_{p} .
\end{aligned}
$$

Proof. The result (i) is obvious from the definition of $M_{r}(f)$. The result (ii) follows by applying Lemma 2.4 to (i). Then

(3.2) THEOREM. Let $f \epsilon L^{p}(0, \infty), 0 \leqslant \gamma<(1 / p)<1,(1 / s)=(1 / p)-\gamma$

$$
\left\|x^{\gamma-1} M_{1}(f)(x)\right\|_{s} \leqslant k\|f\|_{p}
$$


where

$$
k=\left\{\int_{0}^{1} t^{(\gamma-(1 / p)) /(1-\gamma)}|\log (1-t)|^{1 /(1-\gamma)} d t\right\}^{(1-\gamma)} .
$$

Proof. We may assume without loss of generality that $f \geqslant 0$. Then it follows easily by applying Fubini's theorem that

$$
M_{1}(f)(x)=\int_{0}^{x} f(y) \log (x /(x-y)) d y .
$$

The conclusion of the theorem is now easily verified by applying the inequality for homogeneous kernels given in $[5]$, Theorem 1 .

(3.3) THEOREM. Let $f \epsilon L^{p}(0, \infty), 0 \leqslant \gamma \leqslant(1 / p), 1<r<p, \quad(1 / s)$ $=(1 / p)-\gamma$. Then

$$
\left\|x^{\nu-(1 / r)} M_{r}(f)(x)\right\|_{s} \leqslant k_{p, r, \gamma}\|f\|_{p}
$$

where $k_{p, r, \gamma}=r^{\prime}\{(1-\gamma r) /(1-(r / p))\}^{(1 / r)-\gamma}$.

Proof. We may assume that $f \geqslant 0$. Then it follows from Lemma 2.3 (ii) that

$$
n^{n^{\prime \prime \prime}(\mathbf{1} / r)} M_{r}(f)(x) \leqslant r^{\prime}\left(x^{\gamma r-1} \int_{0}^{x} f(y)^{r} d y\right)^{1 / r}
$$

Since $f_{\epsilon}^{r} L^{2 / r}(0, \infty),(p / r)>1$, the integral on the right side may be treated by applying Lemma 2.4. We observe that

$$
x^{\gamma r-1} \int_{0}^{x} f(y)^{r} d y \in L^{\varepsilon, r}(0, \infty), \quad \text { where } \quad(r / s)=(r / p)-r \gamma>0 .
$$

(3.3.1) Note. In vew of result 2.1, the conclusions of 3.1 and 3.2 , with different values for the constants given, may be deduced from 3.3.

4. The function $M_{r}(f): r \geqslant p$. We shall now prove the main mapping theorem in the case $r \geqslant p$ which generalizes the Hardy-Littlewood theorem. Our main proof of the result involves an assumption of the Hardy-Littlewood result. In Note 4.2 we give an alternative proof in the case $p \leqslant r \leqslant 2 p$ which does not depend on the Hardy-Littlewood theorem.

For a proof of the Hardy-Lit lewood result see [3], Theorem 398. A treatment of the conclusion is also contained in [10], p. 31-32, and in $[4], 21.76$.

(4.1) THEOREM. Let $f \in L^{p}(0, \infty), p>1, \quad 0 \leqslant(1 / r) \leqslant(1 / p), \quad(1 / q)$ $=(1 / p)-(1 / r)$. Then

$$
\begin{gathered}
\left\|x^{-1 / r} M_{r}(f)(x)\right\|_{p} \leqslant p^{\prime}\|f\|_{\mu}, \\
\left\|M_{r}(f)\right\|_{q} \leqslant p^{\prime}\|f\|_{p}
\end{gathered}
$$

Proof. It easily follows from the definition of $M_{r}(f)$ that

$$
M_{r}(f)(x) \leqslant x^{1 / r} \sup _{0 \leqslant t<x}\left|(x-t)^{-1} \int_{t}^{x} f(y) d y\right|
$$

so that the result (i) easily follows from the known result involving $M(f)$.

We now turn to the result (ii). The case $r=p$ is rather easy to obtain. For, if we put $r=p$ in Lemma 2.3 (ii), then it follows that

$$
\left\|M_{p}(f)\right\|_{\infty} \leqslant p^{\prime}\|f\|_{p} .
$$

Now from 2.2, or from the definition of $M_{r}(f)$, we easily see that

$$
\begin{aligned}
M_{r}(f)(x) & \leqslant M_{p}(f)(x)^{p / r} M(f)(x)^{1-(p / r)} \\
& \leqslant\left\|M_{p}(f)\right\|_{\infty}^{p / r} M(f)(x)^{p / q} \\
& \leqslant\left(p^{\prime}\right)^{p / r}\|f\|_{p}^{p / r} M(f)(x)^{p / q}
\end{aligned}
$$

Hence the result (ii) follows by applying the Hardy-Littlewood theorem.

(4.2) Note. We shall now give an alternative proof of 4.1 (ii) in the case $p \leqslant r \leqslant 2 p$. As we mentioned in the proof of 4.1 , the case $r=p$ follows from Lemma 2.3. We shall first consider the case $r=2 p$ here. In what follows we assume that $f \geqslant 0$.

From Lemma 2.3 (i) it follows by applying Hölder's inequality that, for $r>1, p \geqslant 1$,

$$
\begin{aligned}
& M_{r}(f)(x)^{r} \leqslant r^{\prime} \int_{0}^{x} f(t)\left((x-t)^{-1} \int_{t}^{x} f(y) d y\right)^{r-1} d t \\
= & r^{\prime} \int_{0}^{x} f(t)\left((x-t)^{-1} \int_{t}^{x} f(y) d y\right)^{(r / p)-1}\left((x-t)^{-1} \int_{t}^{x} f(y) d y\right)^{\left(r / p^{\prime}\right)} d t \\
\leqslant & r^{\prime}\left\{\int_{0}^{x} f(t)^{p}\left((x-t)^{-1} \int_{t}^{x} f(y) d y\right)^{r-p} d t\right\}^{1 / p}\left\{\int_{0}^{x}\left((x-t)^{-1} \int_{t}^{x} f(y) d y\right)^{r} d t\right\}^{1 / p^{\prime}},
\end{aligned}
$$

and it is easily seen on rearranging that

$$
M_{r}(f)(x)^{r} \leqslant\left(r^{\prime}\right)^{p} \int_{0}^{x} f(t)^{p}\left((x-t)^{-1} \int_{t}^{x} f(y) d y\right)^{r-p} d t
$$

Now let $f^{*}$ represent the non-increasing rearrangement of $f$ so that

$$
\left\|f^{*}\right\|_{p}=\|f\|_{p} \quad \text { and } \quad \int_{i}^{x} f(y) d y \leqslant \int_{0}^{x_{-} t} f^{*}(y) d y
$$


for example see [3], and also [10], p. 29-31. Then it follows from 4.2.1 that

$$
\begin{aligned}
\int_{0}^{\infty} M_{2 p}(f)(x)^{2 p} d x & \leqslant\left(\frac{2 p}{2 p-1}\right)^{p} \int_{0}^{\infty} \int_{0}^{x} f(x-t)^{p}\left(t^{-1} \int_{0}^{t} f^{*}(y) d y\right)^{p} d t d x \\
& =\left(\frac{2 p}{2 p-1}\right)^{p}\left\{\int_{0}^{\infty}\left(t^{-1} \int_{0}^{t} f^{*}(y) d y\right)^{p} d t\right\}\left\{\int_{0}^{\infty} f(x)^{p} d x\right\} .
\end{aligned}
$$

Since $2 p /((2 p)-1) \leqslant p^{\prime}$, and since, by Lemma 2.4 , the first integral on the right side of the last inequality is bounded by $p^{\prime}\left\|f^{*}\right\|_{p}=p^{\prime}\|f\|_{p}$, we easily see that

$$
\left\|M_{2 p}(f)\right\|_{2 p} \leqslant p^{\prime}\|f\|_{2} \text {. }
$$

Now suppose that $p<r<2 p$, and let $\alpha$ be given by $(1 / r)=(\alpha / p)+$ $+(1-\alpha) / 2 p$. Then by 2.2 , we have

$$
M_{r}(f) \leqslant M_{p}(f)^{\alpha} M_{2 p}(f)^{1-\alpha}
$$

Hence if $q$ is defined by $(1 / q)=(1-\alpha) / 2 p=(1 / p)-(1 / r)$, we have

$$
\left\|M_{r}(f)\right\|_{q}^{q} \leqslant\left\|M_{p}(f)\right\|_{\infty}^{\alpha q}\left\|M_{2 p}(f)\right\|_{2 p}^{2 p},
$$

and the required conclusion is easily verified from 4.1.1 and 4.2.3.

(4.3) Corollary. Let $f \in L^{p}(0, \infty), p>1, \quad 0 \leqslant \gamma \leqslant(1 / r) \leqslant(1 / p)$ and $(1 / s)=(1 / p)-\gamma$. Then

$$
\left\|x^{\gamma-(1 / r)} M_{r}(f)(x)\right\|_{s} \leqslant p^{\prime}\|f\|_{p} .
$$

Proof. The result is easily verified from (i) and (ii) of 4.1 by applying Hölder's inequality.

5. $M_{r}(f)$ as a function in $L^{p}, 0<p \leqslant 1$. There are certain results involving $M(f)$ as a function in $L^{p}(0, \infty), 0<p \leqslant 1$; seo [2], [10], p. 31-32, and alse [4], 21.80. In the next two theorems we deduce similar results involving $M_{r}(f)$. The Lebesgue measure on $(0, \infty)$ will be denoted by $m$, and we shall write $\log ^{+} x$ for $\log \max (1, x)$.

(5.1) THEOREM. Let $f$ be a non-negative measurable function on $(0, \infty$ such that $f \log ^{+} f \in L^{1}(0, \infty)$. Then for any measurable set $E \subset(0, \infty)$ ther are constants $a$ and $b$ such that

$$
\int_{E} x^{-1 / r} M_{r}(f) \cdot(x) d x \leqslant a \int_{0}^{\infty} f(x) \log ^{+} f(x) d x+b m(E) \quad(r>0) .
$$

(5.2) Theorem. Let $f \epsilon L^{\mathrm{I}}(0 ; \infty)$, let $0<p<1$, and let $E$ be any measurable set in $(0, \infty)$. Then for $r>0$ we have

$$
\int_{E}\left(x^{-1 / r} M_{r}(f)(x)\right)^{p} d x \leqslant(1-p)^{-1} m(E)^{1-p}\left(\int_{0}^{\infty}|f(x)| d x\right)^{p}
$$

The conclusions of Theorems 5.1 and 5.2 are easily obtained from the known inequalities involving $M(f)$ since, as in the proof of Theorem 4.1 , we have

$$
x^{-1 / r} M_{r}(f)(x) \leqslant M(f)(x) .
$$

6. The function $\tilde{N}_{r}(f)$. We shall now obtain a mapping theorem for $\tilde{N}_{r}(f)$ in the case $r \geqslant p$ similar to that given in Theorem 4.1 (ii) for $M_{r}(f)$. We assume throughout this section that $f$ is a non-negative measurable function on $(-\infty, \infty)$.

(6.1) THEOREM. Let $f \epsilon L^{p}(-\infty, \infty), p>1,0 \leqslant(1 / r) \leqslant(1 / p), \quad(1 / q)$ $=(1 / p)-(1 / r)$. Then there is a finite constant $k_{p, r}$ such that

$$
\left\|\tilde{N}_{r}(f)\right\|_{q} \leqslant k_{p, r}\|f\|_{p}
$$

Proof. It is clearly sufficient to prove that

$$
\left(\int_{0}^{\infty} \tilde{N}_{r}(f)(x)^{q} d x\right)^{1 / p} \leqslant k_{p, r}\|f\|_{p}
$$

A similar result involving $\int_{-\infty}^{0} \tilde{N}_{r}(f)(x)^{q} d x$ can be obtained by changing variables and considering $f(-t)$ in place of $f(t)$.

For $r \geqslant p>1$ we have

$$
\begin{aligned}
\tilde{N}_{r}(f)(x)^{r} & =\int_{0}^{\infty}\left(t^{-1} \int_{x-t}^{x} f(y) d y\right)^{r} d t+\int_{0}^{\infty}\left(t^{-1} \int_{x}^{x+t} f(y) d y\right)^{r} d t \\
& \equiv N_{r}^{(1)}(f)(x)^{r}+N_{r}^{(2)}(f)(x)^{r}
\end{aligned}
$$

and on using the inequality $(a+b)^{1 / r} \leqslant a^{1 / r}+b^{1 / r}, a>0, b>0$, we have

$$
\tilde{N}_{r}(f) \leqslant N_{r}^{(1)}(f)+N_{r}^{(2)}(f)
$$

It is now sufficient to consider $N_{r}^{(1)}(f)$ and $N_{r}^{(2)}(f)$. However, the simplest treatment of the two functions is similar to that we shall give in the next section for the $n$-dimensional form of fractional maximal functions. Hence we omit further details here.

7. The $n$-dimensional fractional maximal function. We denote by $E_{n}$ the Euclidean space of dimension $n(n \geqslant 1)$. For $\underline{x}=\left(x_{1}, x_{2}, \ldots, x_{n}\right) \in E_{n}$, we define $|\underline{x}|$ by

$$
|x|^{2}=\left(x_{1}^{2}+x_{2}^{2}+\ldots+x_{n}^{2}\right) .
$$


As usual, $L^{p}\left(E_{n}\right)$ denotes the space of measurable functions on $E_{n}$ such that

$$
\|f\|_{p}=\left(\int_{E_{n}}|f(\underline{x})|^{p} d \underline{d x}\right)^{1 / p}<\infty
$$

The extension to $n$ dimensions of the maximal theorem of Hardy and Littlewood was first given by Calderón and rygmund [1]. We shall now consider the $n$-dimensional form of the operator $N_{r}$. This is defined by

$$
\left.N_{r}(f)(\underline{x})=\int_{0}^{\infty}\left|t^{-n+((n-1) / r)} \int_{|y| \leqslant t} f(\underline{x}-\underline{y}) d \underline{y}\right|^{r} d t\right)^{1 / r} .
$$

(7.2) THeorem. Let $f \epsilon L^{p}\left(E_{n}\right), n \geqslant 1, p>1, r \geqslant p,(1 / q)=(1 / p)-$ $-(1 / r)$. Then there is a finite constant $k_{n, n, r}$ such that

$$
\left\|N_{r}(f)\right\|_{q} \leqslant k_{n, p, r}\|f\|_{p} .
$$

Proof. As in the case involving $M_{r}$ in Theorem 4.1, we prove the theorem by first considering the functions $N_{\infty}(f)$ and $N_{p}(f)$. Since we shall assume the result

$$
\left\|N_{\infty}(f)\right\|_{p} \leqslant k_{n, p}\|f\|_{p}
$$

given in [1], p. 114-116, we need only consider $N_{p}(f)$.

The simplest procedure in the treatment of the $n$-dimensional $N_{p}(f)$ involves the introduction of the non-increasing rearrangement of $f$ onto $(0, \infty)$. As in Note 4.2 we denote this by $f^{*}$. Then we have

$$
\left\|f^{*}\right\|_{p}=\|f\|_{p} \quad \text { and } \quad\left|\int_{|v| \leqslant t} f(\underline{x}-\underline{y}) d \underline{y}\right| \leqslant \int_{0}^{t^{n_{w_{n}}}} f^{*}(y) d y
$$

where $w_{n}$ represents the volume (n-dimensional Lebesgue measure) of the sphere $\left\{\underline{x} \in E_{n}:|\underline{x}| \leqslant 1\right\}$. From this it follows that, for $p>1$,

$$
\begin{aligned}
\left.N_{p}(f) \underline{x}\right) & \leqslant\left\{\int_{0}^{\infty}\left(t^{-n+((n-1) / p)} \int_{0}^{t^{n} w_{n}} f^{*}(y) d y\right)^{p} d t\right\}^{1 / p} \\
& =w_{n}^{1-(1 / p)} n^{-(1 / p)}\left\{\int_{0}^{\infty}\left(t^{-1} \int_{0}^{t} f^{*}(y) d y\right)^{p} d t\right\}^{1 / 2)} \\
& \leqslant p^{\prime} w_{n}^{1-(1 / p)} n^{-(1 / p)}\left\|f^{*}\right\|_{p} .
\end{aligned}
$$

Hence

$$
\left\|N_{p}(f)\right\|_{\infty} \leqslant p^{\prime} w_{n}^{1-(1 / p} \cdot n^{-(1 / p)}\|f\|_{p}
$$

Now for $p<r<\infty$, we have

$$
\begin{aligned}
N_{r}(f)(x)^{r} & \leqslant\left(\sup _{t \geqslant 0}\left|t^{-n} \int_{|\underline{y}| \leqslant t} f(\underline{x}-\underline{y}) d \underline{y}\right|^{r-p}\right)\left(\int_{0}^{\infty}\left|t^{-n+((n-1) / p)} \int_{|\underline{y}| \leqslant t} f(\underline{x}-\underline{y}) d \underline{y}\right|^{p} d t\right. \\
& =N_{\infty}(f)(\underline{x})^{p r / q} N_{p}(f)(\underline{x})^{p}
\end{aligned}
$$

and the conclusion of the theorem is easily verified by applying 7.2.1 and 7.2.3.

(7.3) Remark. In [8] Smith has proved results involving maximal functions defined on more general measure spaces; see also Rauch [7]. We remark here that it is possible to consider fractional maximal functions in this general setting. We simply replace the spheres $\{y:|y| \leqslant t\}$ of 7.1 by suitable spheres in the metric spaces concerned. The proof of the analogue of Theorem 7.2 will then follow on similar lines.

8. Applications to integral operators. The well-known applications of maximal functions include results involving the majorization of harmonic and subharmonic functions; see [2], [7], [8] and [9]. As may be expected, we can also prove some majorization theorems involving fractional maximal functions. In the first theorem of this section we prove such a theorem for certain one-dimensional operators. Majorization theorems involving operators defined on $L^{p}\left(E_{n}\right), n>1$, are not as straightforward as the one-dimensional case. Hence in the multi-dimensional case we consider results involving the Poisson operator only.

(8.1) THeORem. Let the function $c_{a}(t), a>0, t \epsilon(-\infty, \infty)$ be measurable on $(0, \infty) \times(-\infty, \infty)$, and be absolutely continuous in $t$. Suppose that for each fixed number a, we have

(i) $c_{a} \in L^{p^{\prime}}$,

(ii) $|t|^{1 / p} c_{a}(t) \rightarrow 0$ as $|t| \rightarrow \infty$,

(iii) $t c_{a}^{\prime}(t) \epsilon L^{r^{\prime}}$, where $p \geqslant 1$ and $r \geqslant 1$.

Let the operator $C_{a}$ be defined by

$$
C_{a}(f)(x)=\int_{-\infty}^{\infty} c_{a}(t) f(x-t) d t
$$

and let

$$
\tau(a)=\left(\int_{-\infty}^{\infty}\left|t c_{a}^{\prime}(t)\right|^{r^{\prime}} d t\right)^{1 / r^{\prime}}
$$

Then

$$
\sup _{a>0} \tau(a)^{-1}\left|C_{a}(f)(x)\right| \leqslant \tilde{N}_{r}(f)(x) .
$$


Further, for $p>1, r \geqslant p,(1 / q)=(1 / p)-(1 / r)$, there is a constant $k_{p, r}$ such that

$$
\left\|\sup _{a>0} \tau(a)^{-1}\left|C_{a}(f)\right|\right\|_{q} \leqslant k_{p, r}\|f\|_{p} .
$$

Proof. Since $c_{a} \epsilon L^{p^{\prime}}$, it is clear that $C_{a}(f)$ is defined for $f \epsilon L^{p}$ and $a>0$; in fact, $C_{a}(f)$ is continuous on $(-\infty, \infty)$. Now we have

$$
\begin{aligned}
C_{a}(f)(x) & =\int_{-\infty}^{\infty} c_{a}(t)\left(\frac{d}{d t} \int_{x-t}^{x} f(y) d y\right) d t \\
& =\int_{-\infty}^{\infty} \frac{d}{d t}\left(c_{a}(t) \int_{x-t}^{x} f(y) d y\right) d t-\int_{-\infty}^{\infty} c_{a}^{\prime}(t)\left(\int_{x-t}^{\infty} f(y) d y\right) d t .
\end{aligned}
$$

It follows by applying Hölder's inequality that

$$
\left\|c_{a}(t) \int_{x-t}^{x} f(y) d y\right\| \leqslant|t|^{1 / p^{\prime}}\left|c_{a}(t)\right|\|f\|_{p}
$$

and, by condition (ii), this tends to 0 as $|t| \rightarrow \infty$. On asing this result it follows that

$$
C_{a}(f)(x)=-\int_{-\infty}^{\infty} c_{a}^{\prime}(t) \int_{x=t}^{x} f(y) d y d t
$$

Hence by applying Hölder's inequality again, we have

$$
\left|C_{a}(f)(x)\right| \leqslant \tau(a)\left(\int_{-\infty}^{\infty}\left|t^{-1} \int_{x-t}^{x} f(y) d y\right|^{r} d t\right)^{1 / r} .
$$

This clearly proves the first result of the theorem. The second result follows at once from Theorem 6.1.

(8.2) Special cases of Theorem 8.1. We define the one-dimensional form of the Poisson operator $P_{a}, a>0$, and the Weierstrass operator $W_{a}, a>0$, respectively by

$$
\begin{gathered}
P_{a}(f)(x)=\frac{1}{\pi} \int_{-\infty}^{\infty} \frac{a}{a^{2}+(t-x)^{2}} f(t) d t \\
W_{a}(f)(x)=(4 \pi a)^{-1 / 2} \int_{-\infty}^{\infty} e^{-(t-x)^{2} / 4 a} f(t) d t .
\end{gathered}
$$

Then it is easy to verify that the kernels of these operators satisfy the conditions of Theorem 8.1. Further, since

$$
\left\{\int_{-\infty}^{\infty}\left|t \frac{d}{d t}\left(a /\left(a^{2}+t^{2}\right)\right)\right|^{r} d t\right\}^{1 / r^{\prime}}=2 a\left(\int_{-\infty}^{\infty}\left|t^{2} /\left(a^{2}+t^{2}\right)^{2}\right|^{r^{\prime}} d t\right)^{1 / r^{\prime}}=a^{-1 / r} k(r),
$$

and

$$
\left\{\int_{-\infty}^{\infty}\left|t \frac{d}{d t}\left(a^{-1 / 2} e^{-t^{2} / 4 a}\right)\right|^{r^{\prime}} d t\right\}^{1 / r^{\prime}}=a^{-1 / 2 r} k_{0}(r)
$$

it follows from Theorem 8.1 that, for $p>1, r \geqslant p,(1 / q)=(1 / p)-(1 / r)$, there is a constant $k(p, r)$ such that

$$
\begin{aligned}
& \left\|\sup _{a>0} a^{1 / r}\left|P_{a}(f)\right|\right\|_{q} \leqslant k(p, r)\|f\|_{p}, \\
& \left\|\sup _{a>0} a^{1 / 2 r} \mid W_{a}(f)\right\|_{q} \leqslant k(p, r)\|f\|_{p} .
\end{aligned}
$$

(8.3) The n-dimensional form of the Poisson operator. The $n$-dimensional form of the Poisson operator is given by

$$
P_{a}(f)(\underline{x})=c_{n}^{-1} \int_{E_{n}} \frac{a}{\left(a^{2}+|\underline{t}|^{2}\right)^{(n+1) / 2}} f(\underline{x}-\underline{t}) d \underline{t} \quad\left(a>0, \underline{x} \epsilon E_{n}\right),
$$

where $c_{n}=\pi^{(n+1) / 2} / \Gamma\left(\frac{1}{2}(n+1)\right)$.

It is well-known that $\sup \left\{\left|P_{a}(f)(\underline{x})\right|, a>0\right\} \leqslant k N_{\infty}(f)(\underline{x})$, where $k$ is some finite constant; see for example [9], p. 44. We shall now show that

$$
\sup _{a>0} a^{n / r}\left|P_{a}(f)(\underline{x})\right| \leqslant k_{r} N_{r}(f)(\underline{x}) \quad(1<r<\infty) .
$$

Firstly we have

(8.4) Lemma. Let $f \in L^{p}\left(E_{n}\right), n \geqslant 1, p \geqslant 1$. Then for $r>1$, we have

$$
\left(\int_{0}^{\infty} a^{n-1}\left|P_{a}(f)(\underline{x})\right|^{r} d a\right)^{1 / r} \leqslant c_{n}^{-1}\left(1+2^{n+1}\left(2^{(n+r) / r}-1\right)^{-1}\right) N_{r}(f)(\underline{x}) .
$$

Proof. The first part of the argument used here is that given in [9], p. 44. We may clearly assume that $f \geqslant 0$. Then

$$
\begin{aligned}
c_{n} P_{a}(f)(\underline{x}) & =a\left(\int_{|\underline{\mid}| \leqslant a}+\int_{|\underline{t}|>a}\right) f(\underline{x}-\underline{t})\left(a^{2}+|\underline{t}|^{2}\right)^{-(n+1) / 2} d \underline{t} \\
& \leqslant a^{-n} \int_{|\underline{t}| \leqslant a} f(\underline{x}-\underline{t}) d \underline{t}+a \int_{|\underline{t}|>a} f(\underline{x}-\underline{t})|\underline{t}|^{-n-1} d \underline{t} .
\end{aligned}
$$

Now

$$
\begin{aligned}
a \int_{|\underline{t}|>a} f(\underline{x}-\underline{t})|\underline{t}|^{-n-1} d \underline{t} & =a \sum_{m=1}^{\infty} \int_{2^{m-1}} \int_{a<|t| \leqslant 2^{m}} f(\underline{x}-\underline{t})|\underline{t}|^{-n-1} d \underline{t} \\
& \leqslant \sum_{m=1}^{\infty} a\left(2^{m-1} a\right)^{-n-1} \int_{|\underline{t}| \leqslant 2} f(\underline{x}-\underline{t}) d \underline{t} ;
\end{aligned}
$$


hence, for $r<\infty$,

$$
\begin{aligned}
& c_{n}\left(\int_{0}^{\infty} a^{n-1}\left|P_{a}(f)(x)\right|^{r} d a\right)^{1 / r} \\
\leqslant & N_{r}(f)(\underline{x})+2^{n} \sum_{m=1}^{\infty} 2^{1-m_{2}-m(n-1) r r}\left\{\int_{0}^{\infty}\left|\left(2^{m} a\right)^{-n+1 \cdot(n-1) r r} \int_{|t|<2} f(\underline{x}-\underline{t}) d t\right|^{r} d a\right\}^{1 / r} \\
= & N_{r}(f)(\underline{x})+2^{n}\left(\sum_{m=1}^{\infty} 2^{1-m} 2^{-m n / r}\right) N_{r}(f)(\underline{x}),
\end{aligned}
$$

and this gives the required conclusion.

(8.4.1) Note. A result of the form given in 8.4 with. $N_{r}(f)$ replaced by fractional integrals, can be obtained by applying the continuous form of Minkowski's inequality; see [7].

(8.5) THEOREM. Let $f \epsilon L^{p}\left(E_{n}\right), n \geqslant 1, p>1, r \geqslant p,(1 / q)=(1 / p)-$ $-(1 / r)$. Then there are constants $k_{n, r}$ and $k_{n, p, r}$ such that

and

$$
\sup _{a>0} a^{n / r}\left|P_{a}(f)(\underline{x})\right| \leqslant k_{n, r} N_{r}(f)(\underline{x}),
$$

$$
\left\|\sup _{a>0} a^{n / r} \mid P_{a}(f)\right\|\left\|_{a} \leqslant k_{n, p, r}\right\| f \|_{p} .
$$

Proof. We may clearly assume that $f \geqslant 0$. Since the function

$$
a^{n+1} /\left(a^{2}+y^{2}\right)^{(n+1) / 2}=1 /\left(1+(y / a)^{2}\right)^{(n+1) / 2}
$$

is increasing in $a$, it follows that $a^{n} P_{a}(f)(\underline{x})$ is an increasing function of $a$ on $(0, \infty)$. Hence for $\gamma>1$, we have

$$
(\gamma-1)^{-1} a^{n+1-\gamma} P_{a}(f)=a^{n} P_{a}(f) \int_{a}^{\infty} y^{-\gamma} d y \leqslant \int_{a}^{\infty} y^{n-\nu} P_{y}(f) d y,
$$

and it follows by applying Hölder's inequality that

$$
(\gamma-1) a^{n+1-\gamma} P_{a}(f) \leqslant\left(\int_{a}^{\infty} y^{n-1+(1-\gamma) r^{\prime}} d y\right)^{1 / r^{\prime}}\left(\int_{0}^{\infty} y^{n-1} P_{y}(f)^{r} d y\right)^{1 / r}
$$

If we choose $\gamma$ so that we also have $\left(n / r^{\prime}\right)+-1-\gamma<0$, then by applying Lemma 8.4: we easily see that

$$
\sup _{a>0} a^{n / r} P_{a}(f) \leqslant \pi_{r} N_{r}(f) \text {. }
$$

This proves (i). The result (ii) is an immediate consequence of (i) and Theorem 7.2.

(8.5.1) Note. The procedure employed in the proof of Theorem 8.5 can be applied to other special cases. Among these is the $n$-dimensional form of Weierstrass operator with kernel.

$$
(4 \pi a)^{-n / 2} e^{-|t-x|^{2} / 4 a}, \quad a>0 .
$$

\section{References}

[1] A. P. Calderón and A. Zygmund, On the existence of certain singular integrals, Acta Math. 88 (1952), p. 85-139.

[2] G. H. Hardy and J.E. Littlewood, A maximal function for function-theoretic applications, ibidem 54 (1930), p. 81-116.

[3] G. H. Hardy, J. E. Littlewood and G. Polya, Inequalities, Cambridge 1934.

[4] E. Hewitt and K. Stromberg, Real and abstract analysis, Berlin 1965. [5] G. 0. Okikiolu, Bounded linear transformations in $L^{p}$ space, J. London Math. Soc. 41 (1966), p. 407-414.

[6] - On operators which depend on some parameter, Proc. Cambridge Phil. Soc. 63 (1967), p. $359-366$.

[7] H.E. Rauch, Harmonic and analytic functions of several variables and the maximal function of Hardy and Littlewood, Canadian J. Math. 8 (1956), p. 171 - 184.

[8] K. T. Smith, A generalization of an inequality of Hardy and Littlewood, ibidem 8 (1956), p. 157-170.

[9] E. M. Stein and G. Weiss, On the theory of harmonic functions in several variables, Acta Math. 103 (1960), p. 25-62.

[10] A. Zygmund, Trigonometric series, Vol. I, Cambridge 1959.

UNIVERSITY OF EAST ANGLTA

NORWICH, ENGLAND

Reçu par la Rédaction le 18. 12. 1967 